Supporting Information for:

\title{
Enhancing the Electrospinnability of Low Molecular Weight Polymers Using Small Effective Crosslinkers
}

\author{
Xiaoxiao Wang, Christian Pellerin* and C. Geraldine Bazuin* \\ Centre de recherche sur les matériaux auto-assemblés (CRMAA/CSACS), Département de chimie, \\ Université de Montréal, C.P. 6128, succ. Centre-Ville, Montréal (QC), Canada H3C 3J7
}


Additional infrared spectra and specific viscosity-concentration plots
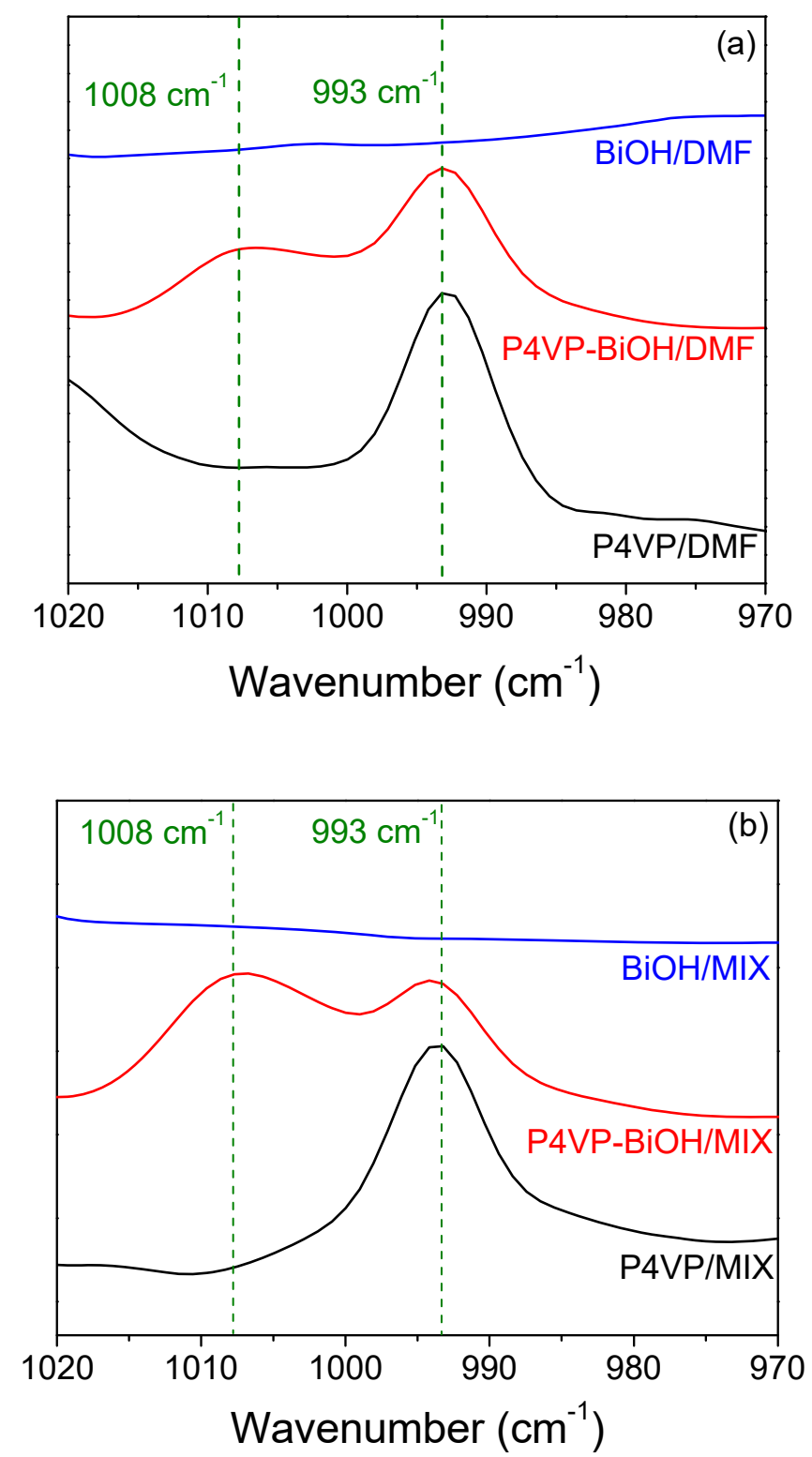

Figure S1. ATR spectra of $10 \mathrm{wt} \% \mathrm{BiOH}, \mathrm{P} 4 \mathrm{VP} 50 \mathrm{k}-\mathrm{BiOH}(20 \mathrm{wt} \% \mathrm{P} 4 \mathrm{VP})$, and pure P4VP (25 wt\% P4VP) in (a) DMF and (b) MIX. The spectrum of pure DMF or MIX, as appropriate, was subtracted from each original spectrum. 
The $993 \mathrm{~cm}^{-1}$ band in the spectrum of pure P4VP in DMF (Fig. S1a) is attributed to a deformation mode of the free pyridine rings. ${ }^{1}$ This band shifts to a higher wavenumber, around $1008 \mathrm{~cm}^{-1}$, when the rings are hydrogen bonded by hydroxyl groups. ${ }^{2}$ Both of these bands are observed in the spectrum of P4VP-BiOH/DMF (Fig. S1a), indicating partial hydrogen-bonded complexation even if DMF is also a hydrogen bond acceptor. Similar spectra are observed for solutions in MIX (Fig. S1b), but the relative intensity of the 1008 $\mathrm{cm}^{-1}$ band is higher, indicating a higher extent of hydrogen bonding in the presence of nitromethane. The relative intensities of these bands can be used to quantify the percentage of hydrogen bonded pyridine rings using Eq. S1:

$$
f_{b}=\frac{I_{b}}{I_{b}+I_{f} / a} \times 100 \%
$$

where $I_{f}$ and $I_{b}$ are the intensities of the 993 and $1008 \mathrm{~cm}^{-1}$ bands, respectively, and $a$ is the ratio of their absorption coefficients. $I_{f}$ and $I_{b}$ were obtained through a second-derivative analysis of the absorbance spectra and $a$ was determined previously to be $0.4{ }^{1}$

The P4VP concentration in Fig. S2 was recalculated as an "apparent P4VP concentration" to consider a possible molecular weight increase of the polymer due to $\mathrm{BiOH}$ hydrogen-bond attachment in the P4VP-BiOH solutions (shown in black). This was done by multiplying each concentration by the factor, where 186.21 and $105.14(\mathrm{~g} / \mathrm{mol})$ are the molecular weights of $\mathrm{BiOH}$ and $\mathrm{P} 4 \mathrm{VP}$ repeat units, respectively, using the $f_{b}$ value for each concentration as determined by IR (as shown in Fig. 1) and assuming that the $\mathrm{BiOH}$ molecules are hydrogen-bonded to P4VP by one end only. In this case, if the effect of $\mathrm{BiOH}$ on viscosity was due only to an increase in apparent P4VP concentration, the corrected data for P4VP-BiOH solutions (black points) would have overlapped with the data for the pure P4VP solutions (red points), which is not the case in either DMF or MIX. 

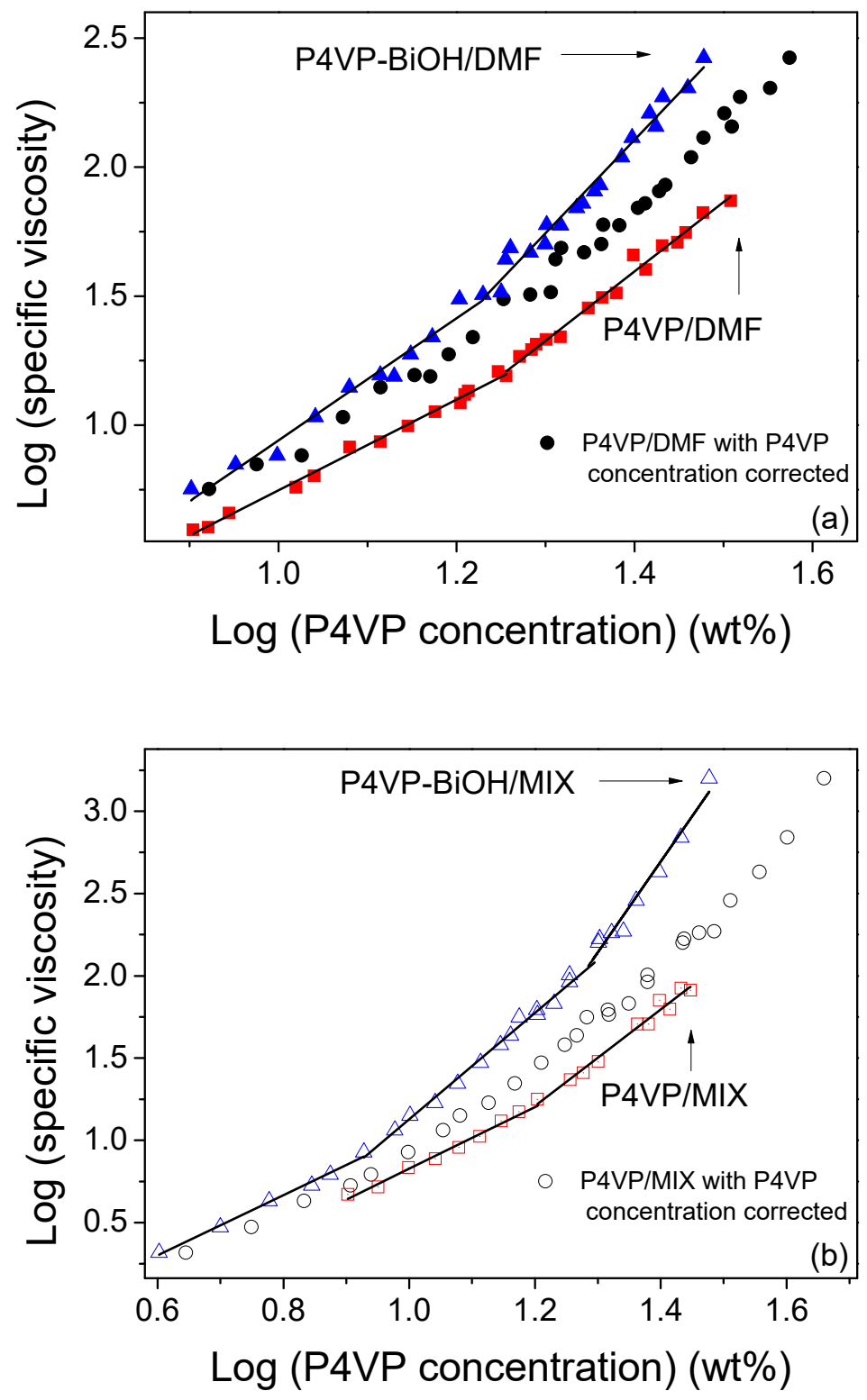

Figure S2. Specific viscosity of P4VP50k (red) and P4VP50k-BiOH (blue) as a function of P4VP concentration for (a) DMF and (b) MIX solutions. The P4VP concentrations for $\mathrm{P} 4 \mathrm{VP}-\mathrm{BiOH}$ (black) were recalculated as apparent P4VP concentrations assuming monofunctionally attached $\mathrm{BiOH}$, in the amounts determined by $f_{b}$ in Fig. 1, as part of the P4VP molecular weight (black data). 

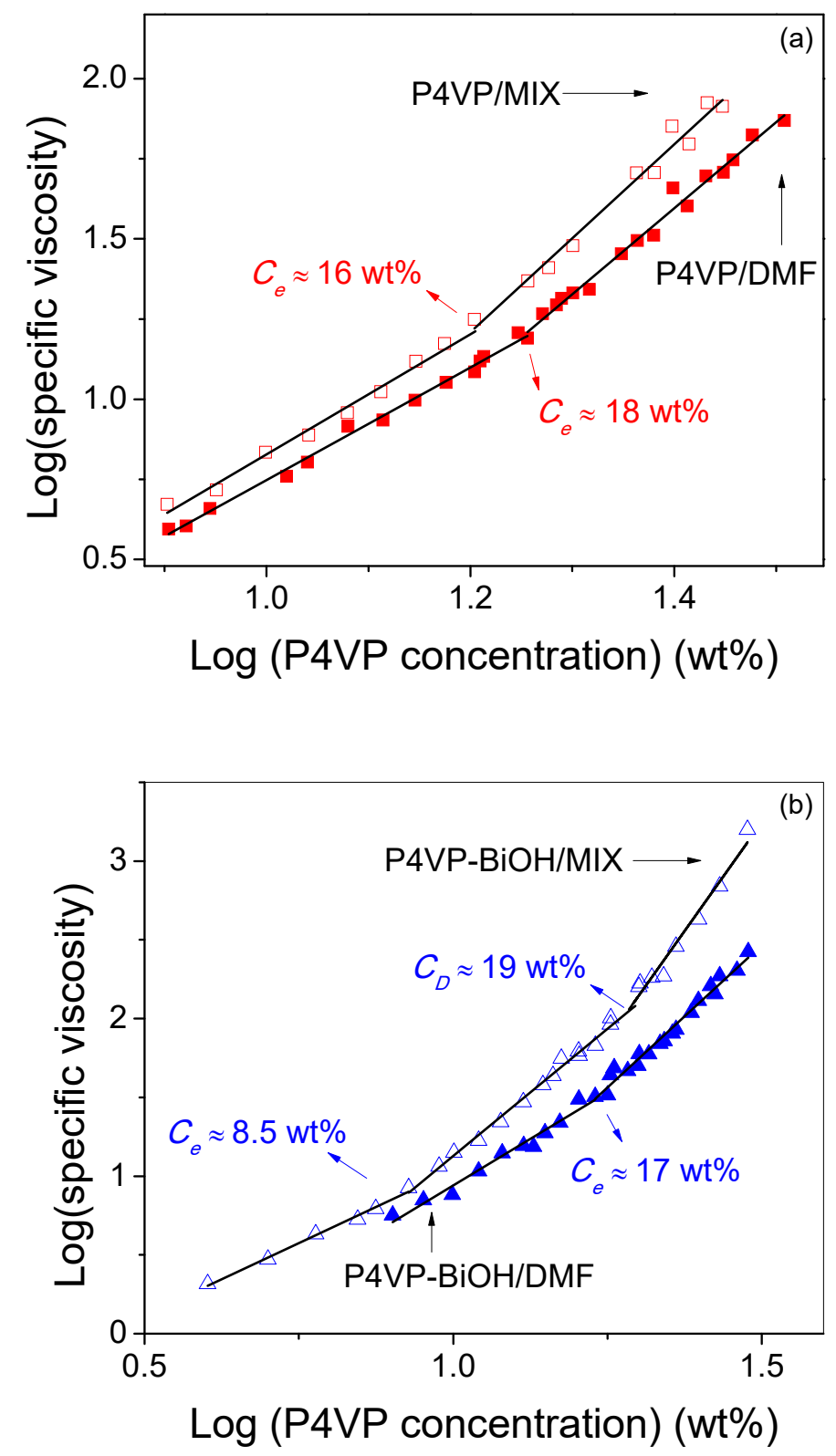

Figure S3. Specific viscosity as a function of P4VP50k concentration for (a) pure P4VP solutions and (b) P4VP-BiOH solutions, showing the effect on viscosity of adding the poor solvent, NM, to DMF (giving MIX). 


\section{Additional SEM images}

As shown in Fig. S4, only droplets are obtained from P4VP/DMF solutions below 27 $w t \%$ concentration. At $27 \mathrm{wt} \%$, very thin fibers form between the droplets with an average fiber length/droplet size of only 3.2. Solutions at $30 \mathrm{wt} \%$ concentration lead to more fibers, whose lengths are 20 to 70 times the average droplet size, including some as long as 20-60 $\mu \mathrm{m}$ (see a low magnification SEM image in Fig. S5). A ratio of about 10 for the average fiber length relative to average droplet size was established in our previous paper as the criterion for defining the formation of beaded fibers. ${ }^{3}$ According to this criterion, $30 \mathrm{wt} \%$ is the critical concentration for fiber formation $\left(c_{f}\right)$ for this system.
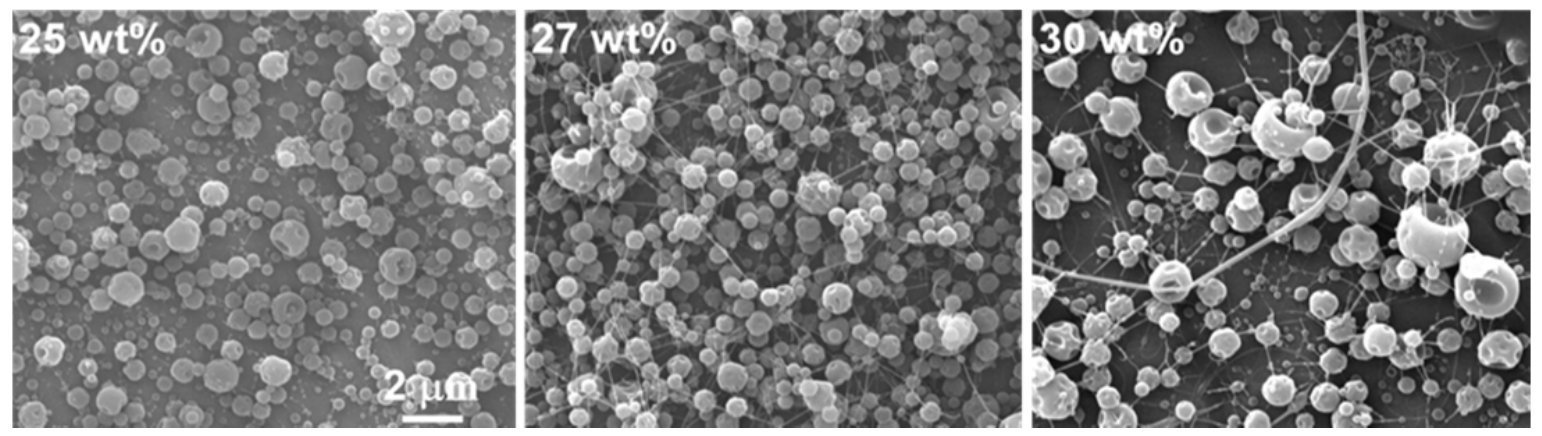

Figure S4. SEM images of electrospun products from P4VP50k/DMF solutions of different P4VP concentrations. The scale bar shown in the first image applies to all images. 


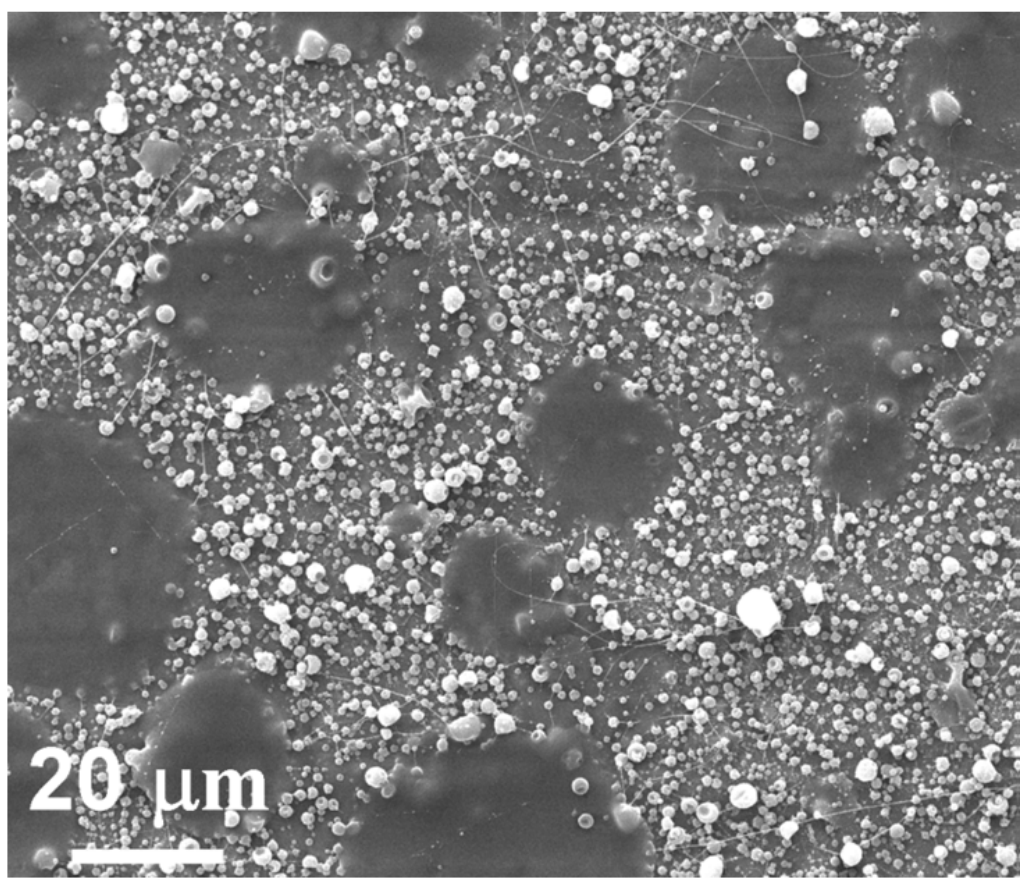

Figure S5. Low magnification SEM image of electrospun products from a $30 \mathrm{wt} \%$ P4VP50k/DMF solution. 
Fig. S6 for P4VP-BiOH/DMF shows that fiber formation begins at $23 \mathrm{wt} \%$ concentration, but they are very thin and short (around $4 \mu \mathrm{m}$; average fiber length/droplet size ratio $\approx 5)$. At $25 \mathrm{wt} \%$, a large amount of longer fibers $(>7 \mu \mathrm{m}$; average fiber length/droplet size ratio $\approx 9$, which is close to the criterion of 10 mentioned above) are obtained, and thus this concentration can be considered as $c_{f}$. Thicker and longer beaded fibers were electrospun from solutions with higher P4VP concentration.
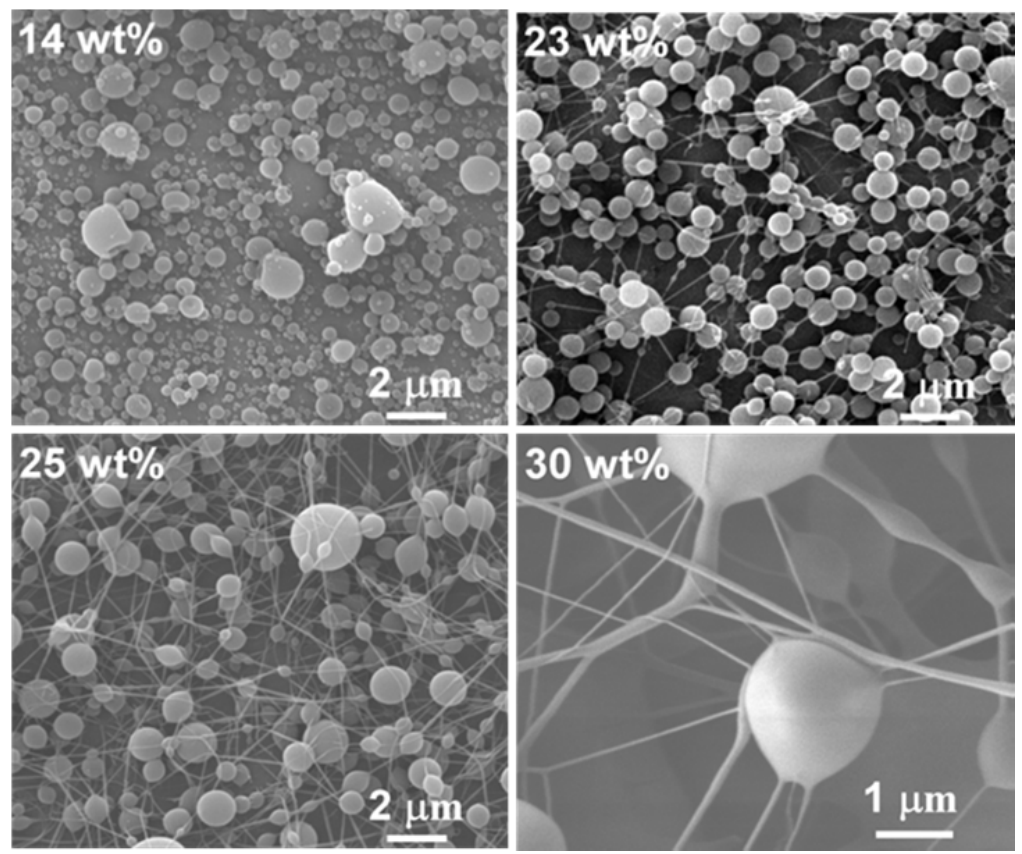

Figure S6. SEM images of electrospun products from P4VP50k-BiOH/DMF with different P4VP concentrations. 
Fig. S7 shows that, when MIX is used as a solvent for pure P4VP, traces of very thin beaded fibers appear at $20 \mathrm{wt} \%$ concentration, but fibers respecting the fiber length/droplet size criterion only start to form at $23 \mathrm{wt} \%$, defining this as $c_{f}$. Above $23 \mathrm{wt} \%$, mixtures of long beaded fibers and droplets were electrospun. As discussed in our previous paper, ${ }^{3}$ the MIX solvent increases the solution viscosity as compared to DMF solutions and thus improves the electrospinnability by facilitating polymer-polymer interactions (which can even lead to gelation in $\mathrm{P} 4 \mathrm{VP} /$ pyridine solutions). ${ }^{4}$
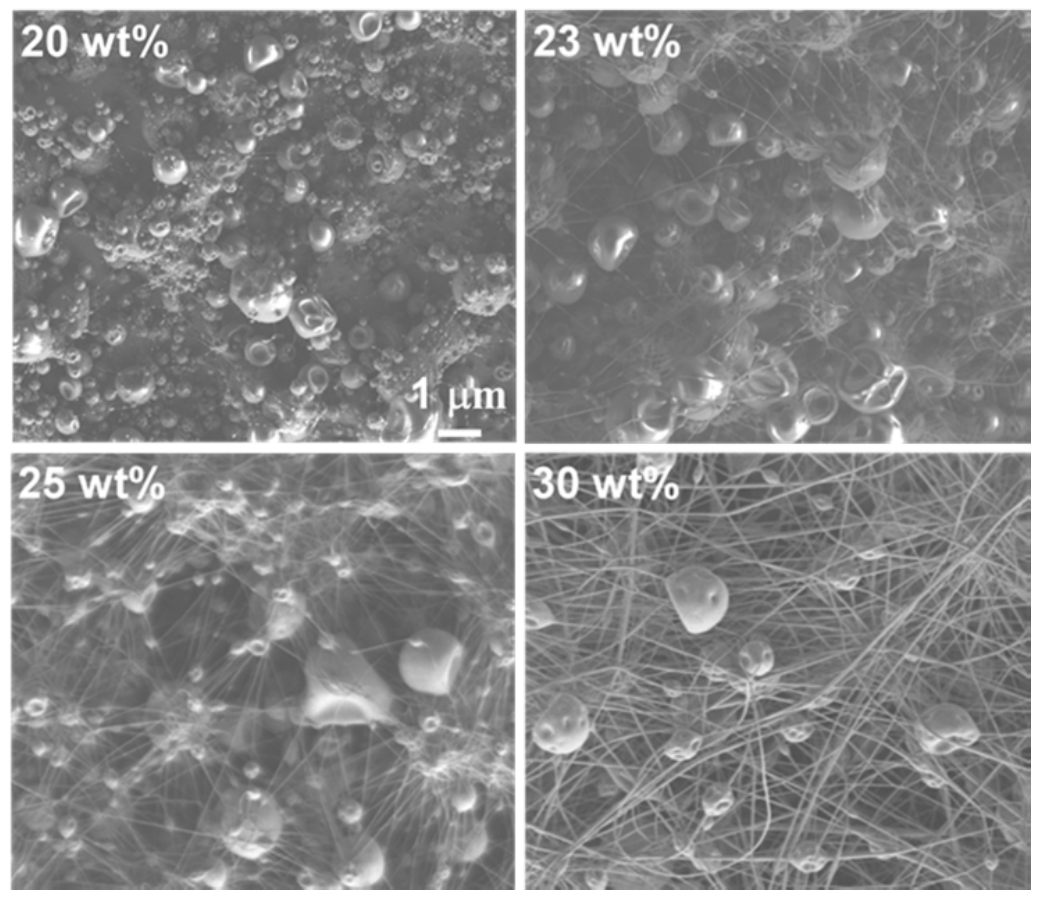

Figure S7. SEM images of electrospun products from P4VP50k/MIX with different P4VP concentrations. The scale bar shown in the first image applies to all images. 
Additional results for $\mathrm{BiOH}$ sublimation

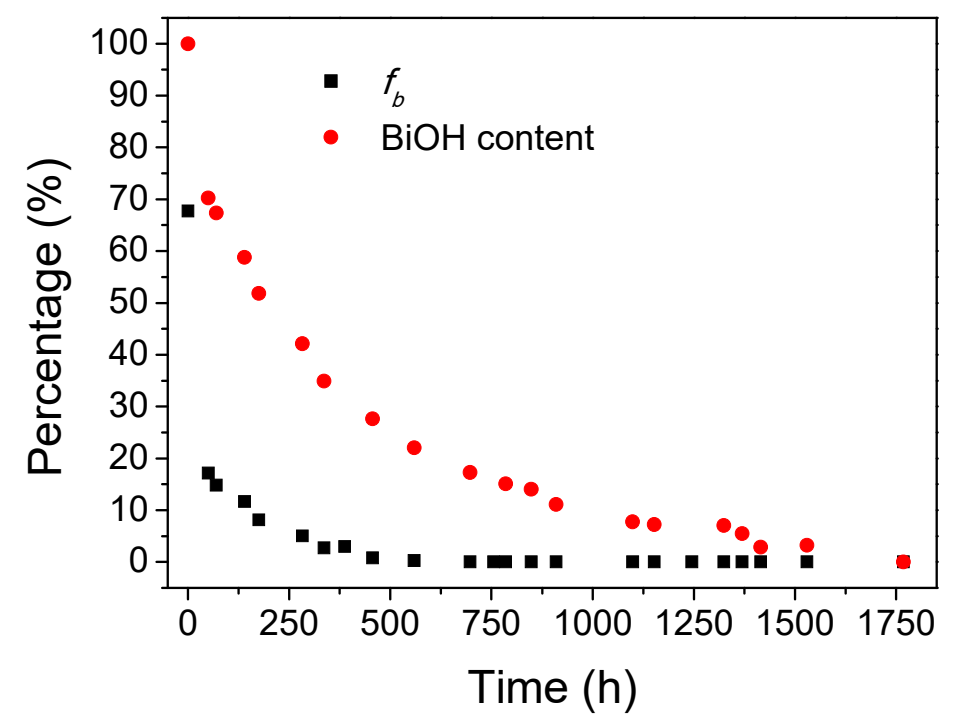

Figure S8. Hydrogen-bonded pyridine rings $\left(f_{b}\right)$ and $\mathrm{BiOH}$ content (expressed as the normalized ratio of the intensity of the $1168 \mathrm{~cm}^{-1} \mathrm{BiOH}$ and $1068 \mathrm{~cm}^{-1}$ P4VP bands) in electrospun fibers of P4VP50k-BiOH/DMF during sublimation at $120^{\circ} \mathrm{C}$. 


\section{Determination of $a$ and $f_{b}$ for P4VP-HBCA}
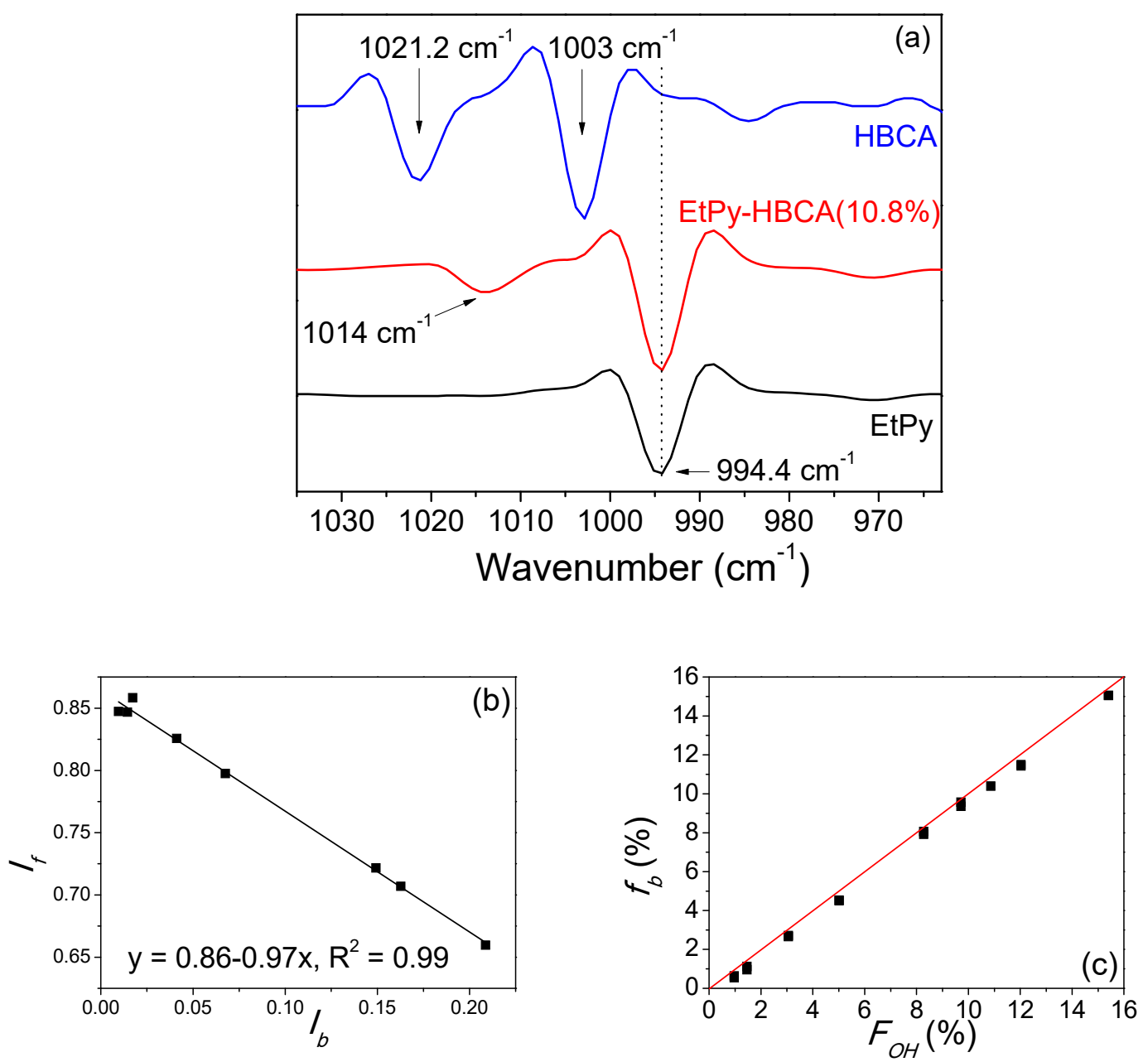

Figure S9. (a) Second-derivative spectra of EtPy, HBCA and the EtPy-HBCA(10.8\%) complex. (b) The intensity, $I_{f}$, of the free pyridine ring band at $994.4 \mathrm{~cm}^{-1}$ versus the intensity, $I_{b}$, of the hydrogen-bonded pyridine ring band at $1014 \mathrm{~cm}^{-1}$, as obtained from the second-derivative spectra of the EtPy-HBCA(0.97-15.4\%) liquid blends. The line represents the least-square fit of the data points. (c) Percentage of hydrogen-bonded pyridine rings, $f_{b}$, as a function of HBCA content, $F_{O H}$, for EtPy-HBCA liquid blends. The red line shows the theoretical values for full complexation of EtPy by HBCA. 
The determination of $a$ for the P4VP-HBCA system was made as described in our previous paper, ${ }^{1}$ by using liquid blends of EtPy and 4-hydroxy-4'-biphenyl carboxylic acid (HBCA) as a model. Fig. S9a shows the second-derivative spectra of EtPy, HBCA, and the complex EtPy-HBCA(10.8\%). Compared with the spectrum of pure HBCA powder, the band at $1003 \mathrm{~cm}^{-1}$ is very weak and the band at $1021 \mathrm{~cm}^{-1}$ is absent in the spectrum of the complex. As for $\mathrm{BiOH}$, the free pyridine band of EtPy at $994.4 \mathrm{~cm}^{-1}$ partially shifts to a higher wavenumber, $1014 \mathrm{~cm}^{-1}$, upon hydrogen-bond complexation of EtPy-HBCA(10.8\%). This position is higher than for $\mathrm{BiOH}\left(1008-1009 \mathrm{~cm}^{-1}\right)$ due to the stronger hydrogen-bond interaction of the carboxylic acid group of HBCA with pyridine.

Because of the limited solubility of HBCA in EtPy, this complex could only be prepared with $F_{O H}$ up to $15.4 \%$. It should be noted that $F_{O H}$ represents the nominal content of both the $\mathrm{OH}$ and $\mathrm{COOH}$ groups present in solution. As shown in Figure S9b, a linear correlation was obtained between the intensity of the second-derivative intensities of the bands at 1014 and $994.4 \mathrm{~cm}^{-1}$ for these complexes. The slope $(k)$ was obtained as 0.97 . According to our previous paper, $k$ should be corrected by a dilution factor to determine the real $a$ using Eq. S2, ${ }^{1}$ in which $\rho_{\text {mix }}$ is the density of the EtPy-HBCA liquid blend and $M$ represents the molecular weight.

$$
a=k \times \frac{\rho_{\text {mix }} M_{E t P y}}{\rho_{E t P y}\left(M_{E t P y}+0.5 M_{H B C A}\right)}
$$

Finally $a$ was determined as 0.56 using $\rho_{\text {mix }}=1.09 \mathrm{~g} / \mathrm{mL}$ (as estimated for EtPy$\operatorname{HBCA}(100 \%)), \rho_{E t P y}=0.942 \mathrm{~g} / \mathrm{mL}, M_{E t P y}=107.15 \mathrm{~g} / \mathrm{mol}$ and $M_{H B C A}=214.22 \mathrm{~g} / \mathrm{mol}$. The $f_{b}$ values agree very well with $\mathrm{F}_{\mathrm{OH}}$, as shown in Fig. S9c.

Fig. S10 shows the second-derivative spectra of the P4VP/DMF, P4VP-HBCA/DMF and HBCA/DMF solutions (20 wt\% P4VP). For P4VP/DMF and P4VP-HBCA/DMF, the P4VP concentration was $20 \mathrm{wt} \%$ and $F_{O H}$ was $100 \%$ for P4VP-HBCA/DMF. Compared 
with the spectrum of HBCA in liquid EtPy, the band at $1003.8 \mathrm{~cm}^{-1}$ remains present in HBCA/DMF and the $1021.2 \mathrm{~cm}^{-1}$ band is shifted to $1015.4 \mathrm{~cm}^{-1}$. As a consequence, it overlaps with the hydrogen-bonded P4VP band at $1014.4 \mathrm{~cm}^{-1}$ and its contribution must be considered in calculating $f_{b}$ in P4VP-HBCA/DMF. We assumed that the intensity ratio (1.23) of the HBCA bands at 1003.8 and $1015.4 \mathrm{~cm}^{-1}$ in HBCA/DMF remained unchanged in P4VP-HBCA/DMF, so that the contribution of the $1015.4 \mathrm{~cm}^{-1}$ band from HBCA to the $1014.4 \mathrm{~cm}^{-1}$ band could be subtracted based on the intensity of $1003.8 \mathrm{~cm}^{-1}$ band. All this allowed the calculation of $f_{b}$ in P4VP-HBCA/DMF (20 wt $\%$ P4VP) as $23.8 \%$.

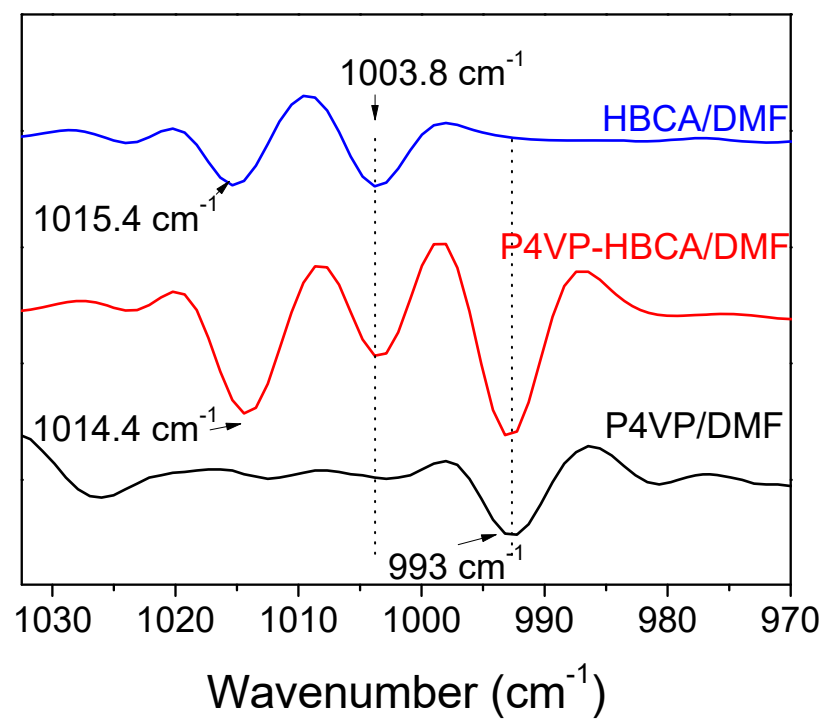

Figure S10. Second-derivative ATR spectra of the solutions of P4VP/DMF (black); P4VPHBCA/DMF (red), $F_{O H}=100 \%$; and HBCA/DMF (blue). Concentration of P4VP $=20 \mathrm{wt} \%$. The DMF spectrum was subtracted from each spectrum. 


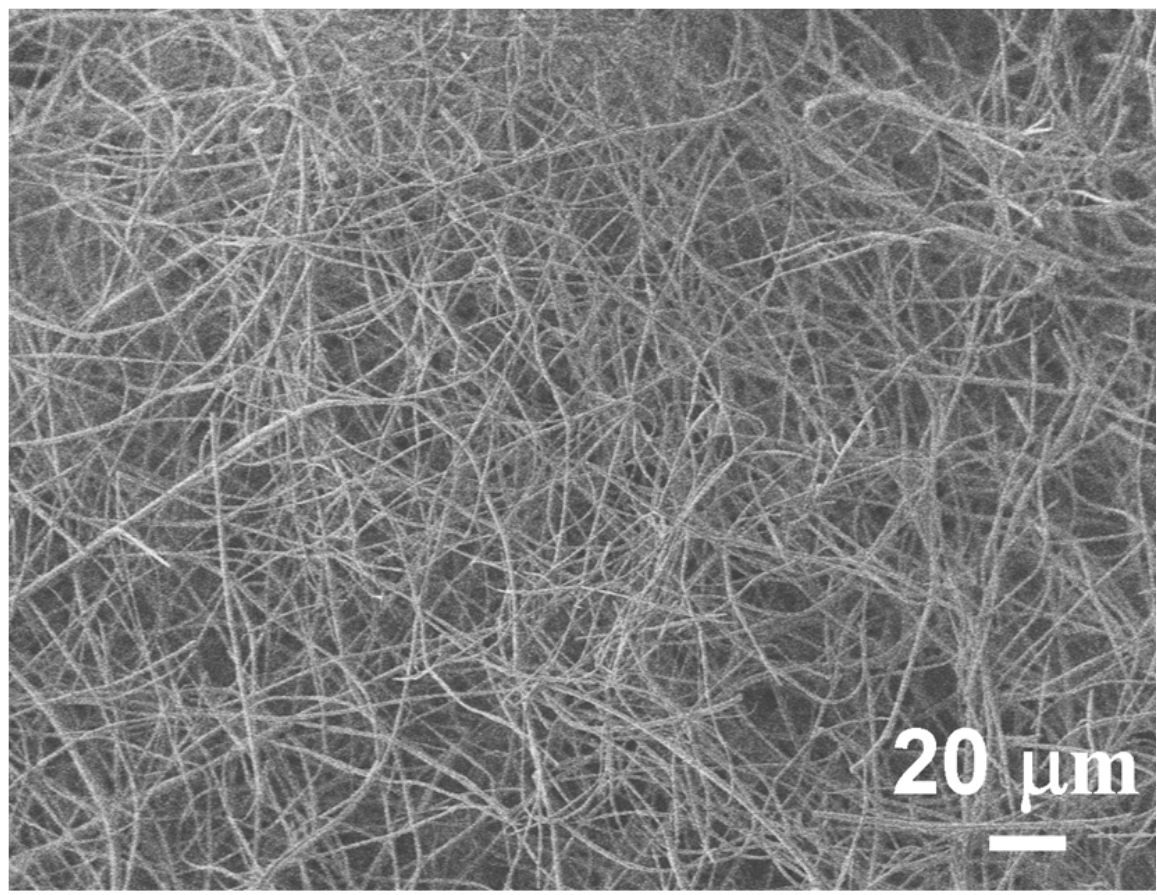

Figure S11. Low magnification SEM image of electrospun products from a $42 \mathrm{wt} \%$ P4VP5k-HBCA/MIX solution.

\section{References}

1. Wang, X.; Bazuin, C. G.; Pellerin, C. Quantitative Analysis of Hydrogen Bonding in Electrospun Fibers of Poly(4-vinyl pyridine)/(4,4' -biphenol) Complexes by ATR Using Liquid Blends as Models. Vib. Spectrosc. 2014, 71, 18-23.

2. Laforgue, A.; Bazuin, C. G.; Prud'homme, R. E. A Study of the Supramolecular Approach in Controlling Diblock Copolymer Nanopatterning and Nanoporosity on Surfaces. Macromolecules 2006, 39, 6473-6482.

3. Wang, X.; Bazuin, C. G.; Pellerin, C. Effect of Small Molecule Hydrogen-Bond Crosslinker and Solvent Power on the Electrospinnability of Poly(4-vinyl pyridine). Polymer 2015, 57, 62-69.

4. Vaganova, E.; Rozenberg, M.; Yitzchaik, S. Multicolor Emission in Poly(4vinylpyridine) Gel. Chem. Mater. 2000, 12, 261-263. 